\title{
BERGMAN OPERATORS FOR ELLIPTIC EQUATIONS IN THREE INDEPENDENT VARIABLES
}

\author{
BY DAVID COLTON
}

Communicated by Fred Brauer, March 25, 1971

Introduction. S. Bergman [1] and I. N. Vekua [7] have both constructed integral operators which map analytic functions of one complex variable onto solutions of the elliptic equation

$$
u_{x x}+u_{y y}+a(x, y) u_{x}+b(x, y) u_{y}+c(x, y) u=0 .
$$

We wish to announce in this note the extension of these results to the three-variable case, i.e. the equation

$$
\begin{aligned}
u_{x x}+u_{y y}+u_{z z}+a(x, y, z) u_{x} & +b(x, y, z) u_{y} \\
& +c(x, y, z) u_{z}+d(x, y, z) u=0
\end{aligned}
$$

where $a, b, c, d$ are real valued entire functions of the (complex) variables $x, y, z$. (With minor modifications we could have assumed only that $a, b, c, d$ are analytic in some ball containing the origin.) Partial results on integral operators for equation (2) (in the special case when $a=b=c=0$ ) have been obtained by Bergman [1], Tjong [6], Colton and Gilbert [4], and Gilbert and Lo [5].

Main results. Let $X=x, Z=\frac{1}{2}(y+i z), Z^{*}=\frac{1}{2}(-y+i z)$. Then equation (2) becomes

$$
\begin{aligned}
U_{X X}-U_{Z Z^{*}} & +A\left(X, Z, Z^{*}\right) U_{X}+B\left(X, Z, Z^{*}\right) U_{Z} \\
& +C\left(X, Z, Z^{*}\right) U_{Z^{*}}+D\left(X, Z, Z^{*}\right) U=0
\end{aligned}
$$

where

$$
\begin{aligned}
& U\left(X, Z, Z^{*}\right)=u(x, y, z), \\
& A\left(X, Z, Z^{*}\right)=a(x, y, z), \\
& B\left(X, Z, Z^{*}\right)=\frac{1}{2}(b(x, y, z)+i c(x, y, z)) \\
& C\left(X, Z, Z^{*}\right)=\frac{1}{2}(-b(x, y, z)+i c(x, y, z)) \\
& D\left(X, Z, Z^{*}\right)=d(x, y, z) .
\end{aligned}
$$

The substitution

AMS 1970 subject classifications. Primary 35A20, 35C15; Secondary 35J15.

Key words and phrases. Integral operators, elliptic equations, analytic functions, complete families. 
(5) $\quad V\left(X, Z, Z^{*}\right)=U\left(X, Z, Z^{*}\right) \exp \left[-\int_{0}^{Z} C\left(X, Z^{\prime}, Z^{*}\right) d Z^{\prime}\right]$

yields the following equation for $V\left(X, Z, Z^{*}\right)$,

$$
\begin{aligned}
V_{X X}-V_{Z Z^{*}}+\tilde{A}\left(X, Z, Z^{*}\right) V_{X} & +\tilde{B}\left(X, Z, Z^{*}\right) V_{Z} \\
& +\tilde{D}\left(X, Z, Z^{*}\right) V=0,
\end{aligned}
$$

where $\tilde{A}, \widetilde{B}, \tilde{D}$ are expressible in terms of the coefficients $A, B, C, D$. Let $U_{0}\left(X, Z, Z^{*}\right)$ be the real valued, entire solution of equation (3) which satisfies the Goursat data $U_{0}\left(X, 0, Z^{*}\right)=U_{0}(X, Z, 0)=1$. Note that in the special case when $D=0$ we can choose $U_{0} \equiv 1$. In the general case when $D \neq 0, U_{0}$ can be constructed via the recursive scheme

$$
\begin{gathered}
U_{0}=1+\lim _{n \rightarrow \infty} W_{n}, \\
W_{n+1}=\int_{0}^{Z} \int_{0}^{Z^{*}}\left(\frac{\partial^{2} W_{n}}{\partial X^{2}}+A \frac{\partial W_{n}}{\partial X}+B \frac{\partial W_{n}}{\partial Z}\right. \\
\left.+C \frac{\partial W_{n}}{\partial Z^{*}}+D W_{n}-D\right) d Z^{\prime} d Z^{* \prime}, \\
W_{0}=0 .
\end{gathered}
$$

By introducing the variables

$$
\begin{aligned}
& \xi_{1}=2 \zeta Z, \\
& \xi_{2}=X+2 \zeta Z, \\
& \xi_{3}=X+2 \zeta^{-1} Z^{*}, \\
& \mu=\frac{1}{2}\left(\xi_{2}+\xi_{3}\right)=X+\zeta Z+\zeta^{-1} Z^{*},
\end{aligned}
$$

where $\zeta$ is a complex variable such that $1-\epsilon<|\zeta|<1+\epsilon, 0<\epsilon<\frac{1}{2}$, we can now state the following theorem. In the theorems which follow "Re" denotes "take the real part" and "Im" denotes "take the imaginary part."

THEOREM 1. Let

$$
E^{*}\left(\xi_{1}, \xi_{2}, \xi_{3}, \zeta, t\right)=\sum_{n=1}^{\infty} t^{2 n} \mu^{n} p^{(n)}\left(\xi_{1}, \xi_{2}, \xi_{3}, \zeta\right)
$$

where 


$$
\begin{aligned}
& p_{1}^{(n+1)}-\frac{1}{2}\left(\tilde{A}^{*}+\widetilde{B}^{*} \zeta\right) p^{(n+1)} \\
& =\frac{1}{2 n+1}\left\{p_{22}^{(n)}+p_{33}^{(n)}-4 p_{13}^{(n)}-2 p_{23}^{(n)}+\left(\tilde{A}^{*}+2 \tilde{B}^{*} \zeta\right) p_{2}^{(n)}\right. \\
& \left.+\widetilde{A}^{*} p_{3}^{(n)}+2 \widetilde{B}^{*} \zeta p_{1}^{(n)}+\widetilde{D}^{*} p^{(n)}\right\}
\end{aligned}
$$

$$
\begin{gathered}
p^{(1)}\left(\xi_{1}, \xi_{2}, \xi_{3}, \zeta\right)=\exp \left[\frac{1}{2} \int_{0}^{\xi_{1}}\left(\tilde{A}^{*}+\tilde{B}^{*} \zeta\right) d \xi_{1}^{\prime}\right] \\
p^{(n+1)}\left(0, \xi_{2}, \xi_{3}, \zeta\right)=0, \quad n=1,2, \cdots, \\
p_{i}^{(n)}=\partial p^{(n)} / \partial \xi_{i}, \quad p_{i j}^{(n)}=\partial^{2} p^{(n)} / \partial \xi_{i} \partial \xi_{j},
\end{gathered}
$$

with $\tilde{A}^{*}\left(\xi_{1}, \xi_{2}, \xi_{3}, \zeta\right)=\tilde{A}\left(X, Z, Z^{*}\right), \tilde{B}^{*}\left(\xi_{1}, \xi_{2}, \xi_{3}, \zeta\right)=\tilde{B}\left(X, Z, Z^{*}\right)$, $\widetilde{D}^{*}\left(\xi_{1}, \xi_{2}, \xi_{3}, \zeta\right)=\widetilde{D}\left(X, Z, Z^{*}\right)$. Then the following is true:

(1) $E^{*}\left(\xi_{1}, \xi_{2}, \xi_{3}, \zeta, t\right)=E\left(X, Z, Z^{*}, \zeta, t\right)$ is regular in $G_{R} \times B \times T$ where $G_{R}=\left\{\left(\xi_{1}, \xi_{2}, \xi_{3}\right):\left|\xi_{i}\right|<R, i=1,2,3\right\}, B=\{\zeta: 1-\epsilon<|\zeta|<1+\epsilon\}$, $T=\{t:|t| \leqq 1\}$, and $R$ is an arbitrarily large positive number.

(2) If $U\left(X, Z, Z^{*}\right)$ is a real valued (for $(x, y, z)$ real) solution of equation (3) which is regular in some neighborhood of the origin, then there exists an analytic function $f(\mu, \zeta)$ which is regular for $\mu$ in some neighborhood of the origin and $|\zeta|<1+\epsilon$, such that locally

$$
U\left(X, Z, Z^{*}\right)=U(0,0,0) U_{0}\left(X, Z, Z^{*}\right)+\operatorname{Re} C_{3}\{f\}
$$

where

$$
\begin{aligned}
C_{3}\{f\}=\frac{1}{2 \pi i} \int_{|\zeta|=1} \int_{-1}^{+1} \exp \left[\int_{0}^{Z} C\left(X, Z^{\prime}, Z^{*}\right) d Z^{\prime}\right] \\
\cdot E\left(X, Z, Z^{*}, \zeta, t\right) f\left(\mu\left(1-t^{2}\right), \zeta\right) \frac{d t}{\left(1-t^{2}\right)^{1 / 2}} \frac{d \zeta}{\zeta} .
\end{aligned}
$$

(3) If

$$
\begin{gathered}
U\left(X, 0, Z^{*}\right)-U(0,0,0)=\sum_{n=0}^{\infty} \sum_{m=0 ; n+m \neq 0}^{\infty} \gamma_{n m} X^{n} Z^{* m} \\
\bar{C}\left(X, Z, Z^{*}\right)=\overline{C\left(X,-Z^{*},-Z\right)}, \quad x, y, z \text { real }
\end{gathered}
$$

then

$$
f(\mu, \zeta)=\sum_{n=0}^{\infty} \sum_{m=0}^{n+1} a_{n m} \frac{\Gamma(n+2)}{\Gamma\left(n+\frac{1}{2}\right) \Gamma\left(\frac{3}{2}\right)} \mu^{n} \zeta^{m}
$$


where

$$
\begin{gathered}
a_{n-1,0}=\gamma_{n 0}, \quad n \geqq 1, \\
a_{n+m-1, m}=\frac{2 n ! m !}{(n+m) !} \gamma_{n m}-\sum_{k=0}^{n-1} \frac{n !}{(n+m) ! k !} \delta_{k m} \gamma_{n-k, 0}, \quad n \geqq 0, m>0, \\
\delta_{k m}=\left(\frac{\partial^{k+m}}{\partial X^{k} \partial Z^{* m}} \exp \left[\int_{0}^{-Z^{*}} \bar{C}\left(X, Z^{\prime}, 0\right) d Z^{\prime}\right]\right)_{X=Z^{*}=0} .
\end{gathered}
$$

(The finite series in equation (16) is omitted when $n=0$.)

The fact that every real valued twice continuously differentiable solution of equation (2) (i.e., a regular solution of equation (3)) can be represented in the form of equation (11) now leads to the following theorem:

Theorem 2. Let $G$ be a bounded, simply connected domain in Euclidean three space, and, for $x, y, z$ real, define

$$
\begin{gathered}
u_{0}(x, y, z)=U_{0}\left(X, Z, Z^{*}\right) \\
u_{2 n, m}(x, y, z)=\operatorname{Re} C_{3}\left\{\mu^{n} \xi^{m}\right\}, \quad 0 \leqq n<\infty, m=0,1, \cdots, n+1, \\
u_{2 n+1, m}(x, y, z)=\operatorname{Im} C_{3}\left\{\mu^{n} \xi^{m}\right\}, \quad 0 \leqq n<\infty, m=0,1, \cdots, n+1 .
\end{gathered}
$$

Then the set $\left\{u_{0}\right\} \cup\left\{u_{n m}\right\}$ is a complete family of solutions for equation (2) in the space of real valued solutions of equation (2) defined in $G$.

Special cases. (a) $A=B=C=0$.

Theorem 3. A ssume $A=B=C=0$, and let

$$
\tilde{E}^{*}\left(\xi_{1}, \xi_{2}, \xi_{3}, \zeta, t\right)=1+\sum_{n=1}^{\infty} t^{2 n} \mu^{n} p^{(n+1)}\left(\xi_{1}, \xi_{2}, \xi_{3}, \zeta\right)
$$

where the $p^{(n)}$ are defined by equation (10) with $\tilde{A}=\widetilde{B}=0$. Then

(1) $\tilde{E}^{*}\left(\xi_{1}, \xi_{2}, \xi_{3}, \zeta, t\right)=\widetilde{E}\left(X, Z, Z^{*}, \zeta, t\right)$ is regular in $G_{R} \times B \times T$.

(2) Every real valued solution $U\left(X, Z, Z^{*}\right)$ of equation (3) which is regular in some neighborhood of the origin can be represented locally in the form

$$
U\left(X, Z, Z^{*}\right)=\operatorname{Re} P_{3}\{f\}
$$

where

$$
\begin{aligned}
& P_{3}\{f\} \\
& \quad=\frac{1}{2 \pi i} \int_{|\zeta|=1} \int_{-1}^{+1} \tilde{E}\left(X, Z, Z^{*}, \zeta, t\right) f\left(\mu\left(1-t^{2}\right), \zeta\right) \frac{d t}{\left(1-t^{2}\right)^{1 / 2}} \frac{d \zeta}{\zeta},
\end{aligned}
$$


and

$$
\begin{gathered}
f(\mu, \zeta)=-\frac{1}{2 \pi} \int_{\gamma^{\prime}} g\left(\mu\left(1-t^{2}\right), \zeta\right) \frac{d t}{t^{2}} \\
g(\mu, \zeta)=2 \frac{\partial}{\partial \mu}\left[\mu \int_{0}^{1} U(t \mu, 0,(1-t) \mu \zeta) d t\right]-U(\mu, 0,0)
\end{gathered}
$$

In equation (21) $\gamma^{\prime}$ is a rectifiable arc joining the points $t=-1$ and $t=+1$ and not passing through the origin.

(b) $A=B=C=D=0$.

In the special case when $A=B=C=D=0$, the operator $P_{3}$ reduces to the well-known Bergman-Whittaker operator $B_{3}[1]$ and equation (22) gives a new inversion formula for the operator $\operatorname{Re} \boldsymbol{B}_{3}$.

Complete proofs of the results stated in this announcement will appear in [2] and [3].

\section{REFERENCES}

1. S. Bergman, Integral operators in the thory of linear partial differential equations, Ergebnisse der Mathematik und ihrer Grenzgebiete, N.F., Heft 23, Springer-Verlag, Berlin, 1961. MR 25 \#5277.

2. D. Colton, Integral operators for elliptic equations in three independent variables. I, Applicable Analysis (to appear).

3. - Integral operators for elliptic equations in three independent variables. II, Applicable Analysis (to appear).

4. D. Colton and R. P. Gilbert, An integral operator approach to Cauchy's problem for $\Delta_{p+2} u(\mathbf{x})+F(\mathbf{x}) u(\mathbf{x})=0$, SIAM J. Math. Anal. 2 (1971), 113-132.

5. R. P. Gilbert and C. Y. Lo, On the approximation of solutions of elliptic partial differential equations in two and three dimensions, SIAM J. Math. Anal. 2 (1971), 17-30.

6. B. L. Tjong, Operators generating solutions of $\Delta_{3} \psi(x, y, z)+F(x, y, z) \psi(x, y, z)=0$ and their properties, Analytic Methods in Math. Phys., Gordon and Breach, New York, 1970.

7. I. N. Vekua, New methods for solving elliptic equations, OGIZ, Moscow, 1948; English transl., Series in Appl. Math., vol. 1, North-Holland, Amsterdam; Interscience, New York, 1967. MR 11, 598; MR 35 \#3243.

INDIANA UNIVERSITY, BLOOMINGTON, INDIANA 47401 